

\title{
L'éducation à la santé ethnographiée dans un quartier populaire: entre conformisme et dissidence
}

Quentin Courcier, Florian Lebreton, Christophe Gibout

\section{To cite this version:}

Quentin Courcier, Florian Lebreton, Christophe Gibout. L'éducation à la santé ethnographiée dans un quartier populaire: entre conformisme et dissidence. Recherches \& éducations, 2021, 22, 10.4000/rechercheseducations.11163 . hal-03157481

\section{HAL Id: hal-03157481 \\ https://hal.science/hal-03157481}

Submitted on 3 Mar 2021

HAL is a multi-disciplinary open access archive for the deposit and dissemination of scientific research documents, whether they are published or not. The documents may come from teaching and research institutions in France or abroad, or from public or private research centers.
L'archive ouverte pluridisciplinaire HAL, est destinée au dépôt et à la diffusion de documents scientifiques de niveau recherche, publiés ou non, émanant des établissements d'enseignement et de recherche français ou étrangers, des laboratoires publics ou privés. 


\title{
Recherches \& éducations
}

$22 \cdot 2021$ :

La pauvreté scolaire

La pauvreté, l'alimentation et les corps

\section{L'éducation à la santé ethnographiée dans un quartier populaire: entre conformisme et dissidence}

\author{
Quentin Courcier, Florian Lebreton et Christophe Gibout \\ https://doi.org/10.4000/rechercheseducations.11163
}

\begin{abstract}
Résumés
Français English

Notre article porte sur le dispositif institutionnel d'éducation à la santé. Dans un quartier populaire de l'est lillois en voie de gentrification, nous mettons en lumière la tension qui existe entre la « santéisation » des consommations alimentaires et des conduites corporelles et les usages sociaux des populations précarisées dont on souhaiterait qu'elles agissent différemment pour leur bien-être. Cette tension, qui est au cœur de ce que l'on peut qualifier de " gouvernementalité des corps » ou de " biopolitique contemporaine » est analysée à travers ses ressorts, les résistances qu'elle engendre mais aussi les rapports de domination qu'elle (re)produit.
\end{abstract}

Our article focuses on the institutional system of health education. In a working-class neighbourhood in the east of Lille (France) engaged in a process of gentrification, we highlight the tension that exists between the "healthization" of food consumption and bodily behavior and the social uses of precarious populations whose well-being we would like to see them act differently. This tension, which is at the heart of what can be described as the "governmentality of bodies" or "contemporary biopolitics", is analyzed through its springs, the resistances it generates but also the relations of domination it (re)produces.

\section{Entrées d'index}

Mots-clés : éducation à la santé, alimentation, gouvernementalité, normes alimentaires, résistances.

Keywords : health education, nutrition, governmentality of bodies, food standards, resistance 


\section{Texte intégral}

\section{L'éducation à la santé ou le gouvernement des corps ?}

L'éducation dans le champ de la santé nous amène à penser son épistémologie en dehors de celle de l'épidémiologie de laboratoire ou de celles des sciences politiques dans ses développements territoriaux ; l'éducation pour la santé est un objet, un champ de recherches aussi bien en sciences de l'éducation qu'en anthropologie et en sociologie qui nous oblige à penser l'épistémologie de l'éducation, du corps et du social.

L'usage de la terminologie foucaldienne n'est donc pas anodin lorsqu'on parle de politiques publiques de santé. Appliquée aux pratiques de gouvernementalité, aux réflexions bioéthiques et à la critique du néolibéralisme, le corps occupe une place centrale dans l'œuvre de Michel Foucault (1961, 2004). La profusion des concepts foucaldiens se retrouvent chez plusieurs auteurs, abordés sous des angles différents ; de l'apparition d'un nouvel hygiénisme (Bourdelais, 2001), à l'intensification de l'intérêt pour la santé publique (Dozon et Fassin, 2001), ou encore à la systématisation des campagnes de prévention (Berlivet, 2015). Les auteurs s'accordent à trouver en cet hygiénisme, un nouveau schéma de biopouvoir. Le corps et ses usages se situent par conséquent au centre d'enjeux de pouvoir car la manière dont les représentations, incorporations et expériences qui en découlent, le place en relation avec les opérations du pouvoir social (Andrews et Silk, 2011).

Cette démarche "santéiste ", comprise au sens d'une "préoccupation constante et intériorisée pour le bien-être et la qualité de la vie » (Vieille Marchiset, 2019, p. 12) se retrouve dans les dispositifs d'éducation à la santé, tels que les programmes nationaux nutrition santé ( $\left.\mathrm{PNNS}^{1}\right)$ aux slogans évocateurs ; " pour votre santé, pratiquez une activité physique régulière (...) pour votre santé, évitez de manger trop gras, trop sucré, trop salé (...) pour votre santé, mangez au moins cinq fruits et légumes par jour » (www.mangerbouger.fr).

Diffusés sur différents canaux de communication, ils conduisent inévitablement à une normalisation des conduites ; par l'utilisation de guides nutritionnels, de recommandations alimentaires et de classifications « d'actifs » et "d'inactifs ». Être en bonne santé suppose donc d'investir dans un/le capital salutaire qui est à comprendre "dans une dimension sanitaire d'ordre culturel, basée sur un «style de vie », et une teneur morale » (Aceti et Vieille Marchiset, 2014), pour être en bonne santé. L'individu est sommé de poursuivre dans son être, de gérer son corps, de préserver ses organes (Memmi et Taïeb, 2009).

Or, comme le montre la tradition sociologique bourdieusienne, le processus institutionnel normatif se confronte avec les cultures corporelles, notamment celles des classes populaires et précaires qui ne peuvent s'y conformer, par des écarts sociaux entre leurs univers symboliques et les habitudes corporelles relatives à leurs conditions matérielles d'existence dominées (Schwartz, 1998).

En effet, la valorisation d'une alimentation saine, que l'on perçoit intuitivement comme étant une alimentation de qualité, est principalement le fait de classes sociales favorisées et/ou sensibilisées à sa cause (Bourdieu, 1979 ; Guthman, 2011). Son corollaire, la lutte contre « la malbouffe » et, plus récemment, les aliments issus de l'industrie agroalimentaire, passent, en partie, par des tentatives d'imposition de besoins et normes alimentaires, qui sont autant de normes sociales, morales ou politiques valorisées par les classes dominantes (Fauquette et Gibout, 2019b)². De même que, la manière dont se présentent les activités physiques au travers des messages de promotion de la santé privilégient des usages sociaux du corps 
davantage adoptés par les classes moyennes et supérieures (sport et santé, activités corporelles douces et / ou de loisirs) (Boltanski, 1971 ; Génolini et Clément, 2010).

En ce sens, les " bonnes pratiques », légitimées via les canaux institutionnels, semblent ignorer et parfois même renforcer certaines inégalités sociales de santé du fait de leur " incorporation » (Fassin, 1996). On distingue ainsi une double injonction directement adressée aux corps populaires : celle du «bien manger » et celle du « bien bouger » (Le Breton, 2010 ; Génolini et Clément, op.cit).

\section{Contexte de la recherche}

L'interrogation au cœur de notre travail est la suivante : tout en affirmant vouloir lutter contre les inégalités sociales et les excès de l'alimentation industrielle associés à des conduites corporelles inappropriées, les acteurs de la promotion de la santé ne reproduisent-ils pas des rapports de domination englués dans un conformisme social?

Ce questionnement est au cœur de la recherche-action que nous avons mené entre 2017 et 2018 à Fives, une « petite ville dans la ville » Lilloise, que ce soit en termes de nombre d'habitants (20 ooo habitants) ou en termes d'infrastructures et institutions diverses (services publics, commerces). Fives est aujourd'hui un quartier "désouvriérisé » (Belbachir, Bienvenu, Délé, Duchaussoy et Rahmania, 2016, p. 324) qui a subi de plein fouet le phénomène de désindustrialisation avec les fermetures de deux usines en son sein, entre 1998 et 2001. Ces événements ont entraîné un cortège de laissés-pour-compte et une précarisation urbaine. Les stigmates perceptibles de cette précarité urbaine se matérialisent par des familles en détresse sociale et sanitaire, par des adolescents en difficulté scolaire, par l'errance de nombreux groupes de jeunes hommes aux alentours des stations de métro, ainsi que par la présence de nombreux commerces de restauration rapide (kébabs, fast-food, etc.).

Or, depuis plusieurs années, il fait l'objet d'un réinvestissement massif des pouvoirs publics qui misent dans la rénovation de son parc immobilier pour deux raisons principales. D’abord, même s'il demeure enclavé par rapport au centre-ville, il est un quartier particulièrement bien desservi en matière de transports en commun : la station de métro Fives se situe à deux arrêts de celui de la gare centrale (LilleFlandres). Ensuite, il est un quartier limitrophe du cœur économique de Lille, la cité administrative et voit l'arrivée de classes moyennes supérieures qui souhaitent accéder à la propriété ou à des logements spacieux, appartements de type 3 ou 4 ou maisons avec jardin, pour des montants qui demeurent inférieurs au reste de la métropole.

Des signes de la vie du quartier donnent à penser que le quartier est en voie de gentrification : l'installation d'un restaurant bio et d'un supermarché participatif et coopératif, l'apparition de La Sécu (une ancienne structure de la sécurité sociale transformée en galerie d'art contemporain) et par le projet Tast In Fives (TIF) qui s'inscrit dans les " nouveaux modes de vie urbain » avec la réhabilitation de l'ancienne usine Fives-Cail-Babcock en « halles gourmandes » composées d'un foodcourt, d'une serre agricole et d'une offre alimentaire « alternative » qui correspond aux aspirations de ces nouvelles classes urbaines moyennes et supérieures.

En somme, le projet TIF entend traiter la pauvreté urbaine mais les documents institutionnels que nous avons vu passer çà et là, entendent " changer les habitudes alimentaires des populations en difficulté dans le quartier afin de leur permettre d'accéder à un mieux-être ». Cet objectif de santé publique qui intègre la variable environnementale n'est que très rarement annoncé comme tel en public. Il s'inscrit, en fait, dans un processus politique de transformation du quartier (post-industriel) en un éco-quartier ou quartier durable, au sens que les pouvoirs publics souhaitent 
lui donner ; en y apportant une nouvelle offre alimentaire et de nouvelles expériences urbanistiques.

Notre ambition ici est de matérialiser le dispositif institutionnel de promotion de la santé, qui met en lumière la tension qui existe entre, d'un côté, la volonté - d'acteurs issus de milieux sociaux stables - de consommer santé et de pratiquer la ville autrement et, d'un autre côté, la question de l'intégration sociale de populations précarisées dont on souhaiterait qu'elles agissent différemment, en termes de consommation alimentaire voire de pratiques corporelles.

Cette tension, qui est au cœur de ce que l'on peut qualifier de " gouvernementalité des corps » ou de « biopolitique contemporaine » est analysée à travers ses ressorts, les rapports de domination qu'elle produit, mais aussi les résistances qu'elle engendre.

\section{Méthodologie}

Notre enquête s'ancre dans une démarche compréhensive au plus proche des réalités sociales vécues par les habitants de Fives. Inconnus dans le quartier ${ }^{3}$, il nous fallait trouver une stratégie d'approche du terrain qui nous permette de nous entretenir avec des personnes issues de différents milieux sociaux de manière à révéler des représentations sociales spécifiques à partir de nos questions de référence :

- Comment les fractions populaires se représentent-elles l'éducation pour la santé ? Y-a-t-il des exclus?

- L'éducation à la santé a-t-elle pénétré l'espace familial et l'espace scolaire ?

- Quelles sont, en outre, les résistances à cette gouvernementalité des corps par et pour le corps (acte alimentaire et pratiques corporelles) et comment s'exprimentelles?

- Y-a-t-il des acteurs individuels qui se font le relais de la promotion de la santé et si oui de quelles manières agissent-t-ils sous couvert de la teneur morale des discours de santé?

De manière à s'imprégner du quartier, nous avons fait le choix d'investir trois espaces (L'association Ephata, la maison de Quartier et l'Avant-Goût de la Cuisine Commune) en nous présentant simultanément comme sociologues et en se faisant bénévoles actifs de manière à légitimer notre approche. Cette méthode nous a permis d'observer une continuité du travail de recherche et d'instaurer une relation de confiance avec les enquêtés sans rupture temporelle avec le terrain :

\section{L'association Ephata de militantisme solidaire.}

Outre de l'aide administrative et un lieu d'accueil, d'écoute et d'entraide pour les plus démunis, cette association gère un " café solidaire » auto-financé par un magasin de vêtement de seconde main situé au rez-de-chaussée et qui accueille des personnes fragilisées par un parcours de rue. Dans notre enquête, elle représente un lieu où peuvent se restaurer les personnes en situation d'extrême précarité ayant ou ayant eu un parcours de rue. Nous nous sommes entretenus avec deux usagers, Michel (59 ans, sans emploi) et Luc (62 ans, sans emploi) ainsi que le président de l'association Yves (54 ans).

\section{La maison de quartier comme lieu de vie intergénérationnel.}


Cet espace associatif est une porte d'entrée à la discussion et aux échanges sur les problématiques du quartier. Il accueille des familles monoparentales, essentiellement des mères et des jeunes issus des milieux populaires et scolarisés dans le quartier. Cinq entretiens individuels ont été menés avec trois femmes bénévoles, Anne (43 ans, intérimaire), Carole ( 56 ans, sans emploi) et Marie (40 ans, sans emploi) et deux éducateurs ; Nadia (47 ans, responsable secteur adulte) et Youssef (33 ans, animateur socio-éducatif). A ces entretiens s'ajoute un entretien collectif (focus-group) qui s'est déroulé dans le cadre du Lieu d'Accueil de Loisirs de Proximité (LALP) avec quatre jeunes scolarisés ou à la recherche d'une formation post-bac : Abdel (15 ans, lycéen), Ibtissem (19 ans, en recherche d'une formation post-bac), Lola (16 ans, lycéenne) et Mohamed (16 ans, Lycéen).

\section{L'avant-goût de la cuisine commune comme nouvel espace collectif du quartier, animé par les acteurs du projet de renouvellement urbain Tast'in Fives.}

Il est, comme son nom l'indique, une cuisine commune préfabriquée (en attente de l'ouverture de la cuisine commune prévue à l'automne 2020) où les associations peuvent venir cuisiner librement (mais sous réservation) et, éventuellement bénéficier de conseils en termes de « bonnes pratiques alimentaires » (i.e. produits bio, locavores, etc., une forme de «frugalité heureuse » qui n'est pas sans rappeler à quel point les normes diététiques des classes dominantes continuent de s'imposer aux classes populaires via une entreprise normative $c f$. Fauquette et Gibout, 2019a ; Turner, 1982). C'était aussi un "food-court » qui a accueilli de nombreux étudiants, des cadres moyens et des professions intellectuelles supérieures à partir du printemps 2018 jusqu'à l'automne 2019. Il était essentiellement fréquenté par des personnes à la «bonne volonté culturelle » (Bourdieu, 1979) qui n'ont pas de relations directes avec les classes populaires. Outre de très régulières observations du dispositif, nous avons pu réserver cet espace pour organiser un focus-group avec Madame C. (24 ans, étudiante en Master Architecture), Monsieur A. (30 ans, chercheur), Monsieur J. (24 ans, étudiant en Master Aménagement du territoire) et Monsieur Y. (54 ans, responsable associatif).

À partir de ces cas particuliers détaillés et contextualisés, nous nous situons au croisement de plusieurs problématiques et en particulier celle de l'emprise d'un environnement de vie, au sens d'une écologie urbaine (Grafmeyer, Joseph, 1990) sur les comportements de santé.

Treize entretiens semi-directifs où s'entremêlent les questions des rapports à l'alimentation et à l'activité physique ont été organisés sous la forme d'entretiens semi-directifs participatifs ; individuels (10), collectifs (3) et enregistrés à l'aide d'un dictaphone.

A titre d'exemple, les questions amenaient les habitants du quartier à s'exprimer d'abord sur les messages de santé publique (Que pensez-vous du message de santé publique : "pour votre santé, mangez au moins 5 fruits et légumes par jour »? Qu'entendez-vous par " bien manger »? Quelle place occupe l'activité physique dans votre quotidien ?) puis réflexives, en ce sens qu'elles favorisaient le récit processuel (Comment trouvez-vous l'offre sportive du quartier ? Par quel(s) moyen(s) trouvez-vous des produits frais ?). Les verbatim retranscrits ne sont pas uniformes et peuvent parfois apparaître comme autant de détours aux yeux des lecteurs. Ceux-ci ne sont en réalité que ce que les enquêté(e)s ont choisi de nous dire (par exemple, les jeunes adolescents ont une interprétation du «bien manger » au sens gustatif alors que d'autres catégories populaires peuvent se représenter cette notion en son sens quantitatif). Ainsi, même si l'on repère des traits significatifs dans 
les pratiques, elles ne sont pas univoques et peuvent même entrer en dissonance avec les individus d'une même catégorie sociale. Ces différences se retrouveront particulièrement dans le genre et la classe d'âge.

Notons aussi que les limites accordées à cet article ne nous permettent pas de développer certaines dimensions importantes qui interviennent dans l'éducation à la santé des Fivois(e)s rencontré(e)s (comme l'analyse concrète de l'action publique ou la présence d'un personnel médical ou paramédical dans l'environnement familial par exemple). Pour simplifier la lecture, nous garderons en filigrane la question du projet de renouveau du quartier. Ce point sera particulièrement traité en dernière partie où nous prêterons une attention toute particulière à la « bonne santé », au sens d'une valorisation de certaines conduites.

Ainsi, à partir d'un entretien collectif et d'un focus réalisé sur un habitant du quartier que nous appellerons l'« entrepreneur du bien-être 4 », nous aurons l'occasion de revenir sur les rapports de pouvoirs qu'il est possible de saisir au travers des modes de consommation et des pratiques corporelles.

\section{"Manger c'est déjà bien » : modes de gestion des corps précarisés dans le quartier populaire}

Devant une maison mitoyenne en briques ; visible depuis la rue, la vitrine d'un magasin de vêtements de seconde main jouxte un escalier étroit. Ce dernier mène à une grande pièce tout en longueur, un peu défraîchie ; un bureau administratif d'un côté, une cuisine et une grande table à manger de l'autre. C'est ainsi que l'Ephata se donne à voir pour le quidam qui y rentre. Transformée en un lieu associatif, l'étage de la maison est un espace où "n'importe qui peut venir se restaurer ou boire un café " (Yves), les usagers peuvent prendre un repas le midi, gratuitement, s'ils n'ont pas les moyens : "j'accepte maximum 3 euros » (Yves).

Un après-midi d'hiver, deux usagers aux visages gonflés et aux lèvres gercées par le froid tentent de se réchauffer autour d'une tasse de café offerte par le responsable de l'association, ils nous racontent leurs parcours de rue : " quand j'étais jeune, je travaillais à la SNCF à Amiens (..) j’ai eu des problèmes, je voulais aider un gars qui était à la rue alors je l'ai logé (...) mais il me frappait et me volait (....) j’ai dû quitter mon boulot parce que j'avais une mauvaise santé et j’ai eu des problèmes avec l'argent. J'ai habité dans un blockhaus [dans le département de La Somme] et là j’ai trouvé une colocation " (Michel). Ce à quoi rajoute un autre enquêté présent ce jourlà, " moi je travaillais pour La Redoute [société de vente par correspondance], je faisais les catalogues et j'étais assez sportif à la sortie du boulot (...) jusqu'au jour où ils m'ont demandé de faire des trucs que je savais pas faire avec les nouvelles technologies de la communication. J'ai tout perdu, même la santé » (Luc).

Les vies des usagers sont toutes constituées de ruptures, parfois cumulées, qui ont occasionné la perte d'un emploi, d'un logement et ce jusqu'à la perte d'un temps collectif et de lieux de rencontres privilégiés : " quand tu perds tout, tu n'as plus de repère, tu ne sais plus trop où aller, ta famille n'est pas là, tu ne sais pas à qui te confier (...) tu vagabondes » (Luc).

Subir une situation de précarité constitue un emploi à temps plein et la gestion de la précarité occupe en permanence autant les personnes en difficulté que les associations et les foyers ; " ça demande tellement d'énergie d'être dans la précarité (...) c'est du temps pour eux avec les différentes démarches et c'est du temps pour moi parce que je dois tout le temps penser à ce que je vais acheter pour qu'ils mangent le midi, entretenir les locaux... » (Yves).

Les usagers rencontrés mobilisent cette énergie quotidiennement dans l'urgence 
d'une vie d'incertitudes : "le quartier est ce qu'il est... avec ces hauts et ces bas (...) pour linstant, je ne me projette pas j'y vis enfin je fais ce que je peux pour survivre plutôt, c'est la débrouille un peu tous les jours » (Luc). Tout se vie momentanément avec des lendemains aléatoires : «on sait pas ce qu'on va devenir demain » (Michel). Dans La Distinction (1979), Pierre Bourdieu identifie ce lien entre précarité et perception de l'avenir, là où le présent devient « la seule philosophie concevable pour ceux qui, comme on dit, n'ont pas d'avenir et qui ont en tout cas peu de choses à attendre de l'avenir » (Bourdieu, op.cit., p. 203). La subsistance entraîne in fine une « temporalité de l'urgence » comme l'identifie plusieurs auteurs dont les recherches contribuent à placer les rapports au temps au centre des socialisations (Millet \& Thin, 2006 ; Darmon, 2007 ; Masy, 2013). La vie au jour le jour qui caractérise les classes populaires n'a pourtant rien d'hédoniste (Hoggart, op.cit.) et les conduites adaptatives qui s'inscrivent dans une logique de subsistance sont, au contraire, la preuve d'une vigilance extrême (Gibout, 2000 ; Guinchard, 2011).

De ce que nous avons observé, cette vigilance n'est pas préventive, en ce sens qu'elle n'intervient pas dans ce que nous comprenons comme la protection de soi. Le corps d'une dizaine d'usagers n'est pas soigné et peu protégé (beaucoup d'usagers observés ont des difficultés à se mouvoir, des blessures visibles, des problèmes d'hygiène dentaire) : "je sais que j'ai des problèmes à marcher, j’ai une cheville en vrac mais j'ai pas envie d'aller voir quelqu'un ça va me prendre du temps et on va me dire fais ci fais ça» (Michel), mais, il doit être maintenu vigilant par sa restauration physique et par le fait de « bien manger », pris en un sens quantitatif : « il faut bien se nourrir quand on peut le faire, lorsque tu dis bien manger pour nous c'est avoir suffisamment à manger (...) quand y a pas ici je vais au «wagon »5, bien manger c'est pour ne pas flancher » (Michel). Ces difficultés peuvent se retranscrire au travers des représentations, par des conduites adaptatives liées au manque de ressources financières. Comme le déplore Yves, «l'acte alimentaire n'est pas un acte quotidien pour tous les habitants de Fives ». Le fait de s'alimenter maintenant peut alors être considérée comme une action protectrice de soi liée à une future impossibilité de se nourrir.

Dès lors, les logiques préventives de la promotion d'une alimentation saine ne peuvent avoir de prises sur ce public. Pourtant nombreux, ils ne semblent pas être conviés aux ateliers culinaires portés par le projet TIF ; sont-ils mis volontairement de côté ? Yves nous répond qu' " on [les responsables de l'association] avait vaguement entendu parler du projet de la cuisine commune mais personne n'est jamais venu sonner à notre porte pour nous proposer, après c'est vrai que nous accueillons une population particulière (...) peu importe on a notre propre cuisine commune ». Ces déclarations laissent sous-entendre que les individus qu'il accueille sont susceptibles de déranger l'ordre public, par leurs postures, leurs caractéristiques corporelles ou leur simple présence perçus comme des dérèglements et pathologies de la ville (Séchet, 2006).

Les nombreux repas partagés dans l'enceinte associative étaient toujours pris en groupe, partagés par une dizaine d'usagers. Ils comportaient tous, une entrée, un plat et un dessert avec un respect « des parts » au sens de la norme nutritionnelle comme le formule Yves : «on essaie d'apporter un maximum de variété dans la constitution des repas, parce que c'est mieux pour la santé déjà d'avoir des légumes, des féculents et viande ou poisson et parce que ce n'est pas parce qu'ils sont pauvres qu'ils n'ont pas le droit d'être exigeants ».

C'est justement au cours de notre entretien avec lui que nous identifions précisément l'interprétation qu'il pouvait se faire du « bien manger » : «bien manger, je l'entends dans le sens de la qualité (...) pour moi ce serait déjà avoir accès à des bons produits, mais je peux pas me permettre financièrement d'aller à la boucherie, chez le primeur pour préparer les repas c'est impossible (...) je suis contraint d'acheter des aliments premiers prix, attention, ça ne veut pas dire qu'ils tolèrent les fruits et légumes moches, bien au contraire, ils sont conscients de ça et ils veulent des 
produits de qualité ». En pensant à travers son rôle associatif, il nous révèle l'effet culpabilisateur que peut avoir sur lui et sur les usagers la notion de « bien manger » et l'impossibilité pour ses usagers d'avoir accès à une nourriture qualitative entendue au sens des politiques nutritionnelles : "il m'est arrivé d'avoir des remarques sur ce que j'achetais "oh c'est du premier prix », " oh les fruits sont moches ». Ce n'est pas facile parce qu'il ne faut pas penser qu'ils se contentent de manger en quantité suffisante mais ils ont aussi besoin de sentir qu'ils mangent bien ». En effet, même si les travaux sociologiques donnent à voir l'importance du caractère quantitatif dans les manières de s'alimenter pour les catégories dîtes précaires (Corbeau et Poulain, 2002 ; Grassler et al., 2019), il n'en demeure pas moins que le désir qualitatif de nourriture est présent chez certains, comme Luc qui nous dit : "on aime bien manger, des produits frais, une bonne viande, même si c'est pas souvent ».

Bien que partagés, les repas ne s'étendent que très rarement dans la durée, seuls quelques usagers restent discuter. De nos entretiens ressortent toujours des contraintes temporelles liées à d'autres choses à faire et toujours gardées sous silence. Luc et Michel restent, souvent, et nous en profitons pour échanger sur leurs représentations de l'activité corporelle entendue au sens d'une activité physique quotidiennement réalisée. Un peu plus jeunes, les deux faisaient du vélo, marchaient sans objectif particulier, aujourd'hui, loin d'être une errance (Gibout, 2000), l'activité physique qu'ils pratiquent est une marche réalisée sous contrainte : le corps se déplace pour rencontrer, ou au contraire, éviter certaines personnes bienfaisantes (donateurs), utiles (travailleurs sociaux) ou perçues comme dangereuses (bandes de jeunes, junkies), pour Luc : « rester dans la rue c'est dangereux, tu croises toujours des gens un peu bizarres (...) j'aime pas trop rester au même endroit ».

La marche fait encore partie du quotidien de Michel : " de toute façon, je marche tout le temps, avant je faisais un peu de vélo aussi (...) je vais pas payer le bus (...) et puis y a d'autres raisons, vraiment rester trop longtemps dans la rue au même endroit c'est pas bon (...) ça fait tellement longtemps que je n'ai pas marché sans objectif, rien que pour le plaisir, dans la nature, je le faisais quand je vivais à Amiens dans les hortillonnages... ».

Les corps déjà fragilisés par un parcours de rue ne peuvent plus se livrer de manière ludique ou récréative à des pratiques physiques. L'emprise urbaine est perçue d'autant plus négativement que le quartier populaire n'offre pas de répit en termes de précarité des abris, d'insécurité perçue ou encore de contraintes de mobilité pour échapper à de multiples pressions (contrôle de police, stigmatisation ou violences par des autochtones, effets climatiques sur un corps faible et mal protégé, etc.).

Les résistances populaires face aux différentes politiques des corps ont déjà fait l'objet de travaux sociologiques, notamment ceux de Sylvia Faure (2008) qui analyse les politiques du corps visant les milieux populaires. La notion de bonne santé au long terme, présente dans les campagnes de prévention de santé, dans les recommandations alimentaires et des modes de vie actifs reste étrangère aux personnes qui vivent les situations d'extrême précarité. Les rapports au temps, à l'alimentation et au corps deviennent le produit direct du manque de ressources de personnes maintenues à l'écart des normes sanitaires, et même sociales, pendant une longue période. Dès lors, elles ne partagent plus les mêmes codes sociaux que de nombreux interlocuteurs et sont mises à l'écart de l'accès à la "bonne santé » pourtant indivisible de la question sociale.

\section{« Gagner son pain à la sueur de son front » : pratiques alimentaires et corporelles des mères du milieu}




\section{populaire}

Notre enquête se prolonge dans un centre social qui porte le nom d'un ancien ministre lillois, socialiste et populaire : Roger Salengro. Il accueille aussi bien des jeunes adolescents que des femmes qui se caractérisent par de faibles ressources financières et qui sont à la tête de familles monoparentales. Nous les appelons « mères du quartier » (à la suite de leur accord), en ce sens qu'elles ne font pas de différence entre leurs enfants et ceux des autres. Comme Carole l'exprime si bien : "nos enfants sont les enfants du quartier».

Dans ce centre social, l'éducation à la santé se concentre essentiellement sur des actions éphémères autour de la prévention des addictions et à destination d'un public essentiellement masculin, selon Nadia : "Le fléau ici à Lille-Fives, c'est surtout les produits illicites, les dealers, la drogue donc on essaye de sensibiliser là-dessus pour éviter que les gamins tombent dedans ", mais globalement, "le centre n'a pas vocation à tenir un rôle éducatif sur la santé, c'est pas notre rôle, c'est plutôt celui des mères de famille ou de l'école ».

L'éducation à la santé par l'institution scolaire apparaît au cours du XVIIe siècle. Ces objets de travail sont liés à une conception biomédicale de la santé qui va s'étendre de la préservation de la collectivité face aux épidémies jusqu'aux mesures de régulation des comportements sanitaires et des déviances de la norme physique, psychique et morale (Parayre, 2010). De même que, la place de la famille dans les problématiques de santé fait état d'un fonctionnement familial qui propulse - depuis plusieurs générations - les femmes (mères et filles) comme responsables du travail domestique de santé (Cresson, 1997). Comme le note Gilles Vieille-Marchiset, cette justification repose sur la naturalisation des stéréotypes de sexe : "Traditions (ça a toujours été comme ça), habiletés (elles sont plus compétentes), habitudes (on fonctionne comme ça dans notre famille), sans prise de conscience (on n’y a jamais pensé) » (Vieille Marchiset, op.cit., p. 135).

Cette éducation se retranscrit au travers des représentations du « bien manger » des mères qui, par des conduites adaptatives liées au manque de ressources financières, veillent à ce que la famille ne manque de rien pour « être en bonne santé ». Notion intériorisée au sens de ne pas tomber malade : «les signes de la maladie n'est pas ou très peu liée à liidée de prévention ou d'entretien du corps ou de maintien d'un équilibre physiologique mais davantage à celle de surveillance, de protection ou de renforcement du corps face aux menaces externes. » (Thin, 1997, pp. 34-35).

L'idée est d'apporter à la famille une nourriture consistante et le plus souvent carnée, quitte à sortir du quartier pour trouver des commerçants charitables ou pratiquant des prix encore moins onéreux : "déjà, il faut de la viande parce que ça rend fort, ici, difficile d'en trouver de la bonne alors je me rends à Wazemmes chez un boucher que je connais bien pour avoir des bons prix et de la bonne viande » (Carole); "s'ils ne mangent pas suffisamment, ils vont tomber malades alors on se débrouille » (Anne).

Cette peur traditionnelle dans les milieux populaires et ouvriers est relatif au " caractère essentiel, vital de la nourriture pour ceux qui n'ont pas toujours été (historiquement) assurés de leur repas et qui, pour une partie d'entre eux aujourd'hui, renouent avec le risque et la peur de ne rien avoir à " mettre dans l'assiette » (Thin, op.cit., p. 27). à travers d'expressions imagées propres au monde ouvrier : " «se battre pour son beefsteak », "gagner sa croûte », "gagner son pain à la sueur de son front »

Pour Carole qui a connu ce monde ouvrier par ses liens familiaux, les repas étaient toujours bien garnis, riches et conviviaux : "à l'époque de l'usine, il fallait avoir une santé de fer avec des repas qui tiennent au corps (...) à chaque repas du soir il y avait des pommes de terres, de la sauce, de la viande (...) on mangeait tous ensemble, j'essaie de garder ça aujourd'hui ». On distingue ici la ritualisation du 
repas qui participe à l'incorporation de dispositions spécifiques et en particulier à l'intériorisation d'un éthos (Boltanski, 1971 ; Bourdieu, op.cit.) populaire. Pour Pierre Bourdieu (1987), les dispositions sociales, les habitus au sens des structures mentales à travers lesquelles l'acteur du monde social appréhende son monde sont pour l'essentiel le produit de l'intériorisation des structures de ce monde social. Cette orientation sociologique en lien avec l'alimentation précise que cette représentation du « bien manger » - c'est-à-dire manger suffisamment - reste l'un des traits traditionnellement significatifs des familles ouvrières et populaires (Dupuy et Poulain, 2008, p. 263). Cependant, d'autres données pointent aussi une certaine proximité avec les recommandations nutritionnelles.

Par exemple, avec un plus jeune âge et des biographies différentes de celle de Carole, Anne et Marie considèrent que « bien manger » correspond également à la variété et à l'équilibre qu'on peut retrouver dans l'assiette ; "pour moi bien manger, c'est manger varié, avec des fruits, des légumes »; "bien manger, c'est manger équilibré ». Seulement, même s'il demeure des réseaux d'entraide alimentaire identifiables à l'échelle du quartier, l'emprise d'un système socio-écologique complexe et de la précarité surplombent les volontés et entrainent la culpabilité : " franchement trouver un primeur à un bon prix c'est difficile, j'avoue qu'il n'y a pas toujours des fruits ou des légumes à table. Financièrement je ne peux pas me permettre » (Anne) ; " je fais ce que je peux pour quills soient bien et oui c'est vrai des fois je me sacrifie, je mange pas ou moins » (Marie).

Dès lors, les activités physiques et sportives qui participent au « maintien » ou à «la préservation » de la bonne santé semblent bien loin des préoccupations quotidiennes des mères du quartier. Carole ne se sent pas de participer à quelconques activités physiques, elle a un pacemaker et ne voit pas l'intérêt de pratiquer une activité physique si ce n'est " pour être encore plus fatiguée que je ne le suis actuellement ». Anne met en exergue les contraintes organisationnelles vécues dans le quartier en évoquant sa participation régulière aux activités de renforcement musculaire qui étaient proposées dans le cadre du secteur adulte du centre l'année dernière : " beaucoup de femmes du centre faisaient du sport ensemble, ça faisait de la cohésion entre nous, mes filles participaient avec moi ». Elle regrette que l'éducateur sportif soit parti. Elle déplore aussi que le dispositif « Sport Femme Famille », mis en place dans le cadre de la politique de la ville, soit si peu opérant dans le quartier en raison du manque d'éducateur et des horaires qui, selon elle, ne correspondent pas aux emplois du temps de nombreuses mères esseulées. Le manque d'opportunités de pratiques corporelles gratuites est souligné, de même que le fait que les équipements sportifs du quartier restent peu entretenus pour être utilisés dans des conditions favorables à une pratique sportive hors d'un cadre associatif.

Peu touchées par l'action publique de la promotion de la santé, elles se sentent pourtant concernées et essaient d'éduquer à la santé, à leur façon, au travers d'échanges informels avec les jeunes de la maison de quartier : " on leur dit qu'il faut se bouger plutôt que de rester au LALP jouer à la PlayStation $\AA$ " (Anne) ; "les jeunes d'aujourd'hui bougent moins qu'avant il faut les motiver les jeunes (...) en vrai ils n'ont pas beaucoup d'activités sportives à faire » (Marie).

Nous pouvons conclure que les situations vécues laissent peu de place à l'éducation à la santé par l'alimentation et l'activité physique. Parce que les pratiques familiales sont le résultat de logiques familiales incorporées et d'usages sociaux appropriés, les mères rencontrées mettent en œuvre des pratiques plus ou moins proches des attentes et des exigences portées par la promotion de la santé en fonction de leurs ressources culturelles, sociales, économiques et aux contraintes environnementales du quartier (accessibilité des équipements sportifs par exemple). On distingue toujours l'écart dans les discours entre l'homéostasie recherchée par le discours dominant d'entretien et de production d'un état de santé et la conception de la " bonne santé » dans les milieux populaires qui nous apparaît souvent comme l'absence de maladie. 


\section{"Ça compte salade, tomates, oignons dans le kebab? " : la dérision comme contournement habile de la norme alimentaire}

Pour aller plus loin, nous avons souhaité nous poser la question de la pauvreté comme résistance en soi. C'est-à-dire comment les programmes de promotion de la santé exercent un pouvoir qui invisibilise les rapports de force au sein desquels s'exercent une injonction au bien-être. En effet, Il n'est pas exclu de considérer que les pauvretés peuvent aussi se muer en subversion. Refuser l'ordre politique ou économique reviendrait à instituer le risque et le défi, d'un refus d'adhérer ou de se soumettre à une injonction.

Si les programmes de promotion de la santé par les activités physiques ciblent particulièrement les enfants, deux partis-pris s'articulent. D'une part, il s'agit d'agir au plus jeune âge pour créer des habitudes de vie. D'autre part, les enfants peuvent être des ambassadeurs auprès des parents pour avoir une activité physique régulière. Ainsi, en partant du point de vue d'adolescents pour comprendre les processus en jeu dans les pratiques alimentaires et corporelles peut nous aider à apporter des éléments de compréhension au sens qu'ils accordent à ces pratiques, au regard de leur vie quotidienne. Avec l'adoption de ce regard interactionniste, nous ne négligeons pas pour autant le poids des structures sociales sur ces individus.

Pierre Bourdieu (1979) remet en cause l'approche subjectiviste du goût au profit d'une approche parfois qualifiée de déterministe qui s'appuie sur l'idée que les goûts proviennent d'un héritage socio-culturel. C'est par ces habitudes et routines alimentaires que se créent des habitus culturels, ici alimentaires, "c'est-à-dire, une disposition sociétalement inculquée, source d'actions, de perceptions, de comportements et d'appréciations. »(Koch, 2013, p. 45). Pour les sociologues de l'alimentation, l'expression de préférences, de goûts et les manières de manger sont autant d'héritages et de transmissions auxquels sont attribués des significations symboliques les (re)liants à leurs appartenances familiales, sociales et culturelles (Corbeau \& Poulain, 2002 ; Dupuy, Ibid., pp. 80-94).

Le goût se révèle donc comme une pratique incarnée qui exige un espace social et physique pour sa performance : " elle est jouée par le corps et s'y inscrit » (Dupuy, Ibid., pp. 80-94). De cette façon, nous souhaitons mettre en relief non pas ce qu'est le goût, mais plutôt le goût comme modalité de l'habitus, comme disposition et principe génératif de perceptions, comportements et d'actions mises en scène dans le quartier et par les jeunes rencontrés. Cette dimension symbolique, voire identitaire, se retranscrit dans nos entretiens à travers un mode de consommation alimentaire caractéristique de l'offre alimentaire locale. La notion de « bien manger » est associée à un mode de consommation qui sert simultanément le plaisir gustatif tout en renforçant le sentiment d'appartenance au groupe : «là-bas c'est notre repère, c'est le meilleur kebab de Fives » (Abdel), "à Fives y a le choix en kebab mais y en a quand même qui sont au-dessus [du lot] » (Mohamed). Pour Abdel : "c'est le fief et tout le monde se connaît. Les grands nous amènent là-bas, après on y va, après on emmène les petits ».

Il s'agit bien là d'un marquage territorial et identitaire qui se manifeste, entre autres, par une utilisation de la « restauration rapide » comme un espace de socialisation alimentaire, mais aussi, de socialisation globale correspondant à un espace socioculturel spécifique ainsi qu'à un enjeu d'investissement identitaire local pour les personnes issues du quartier.

Depuis la mise en place des Plans nationaux nutrition santé (2001), le goût vu comme pédagogie, le goût vu sous sa forme sanitaire attend des jeunes qu'ils participent à 
une éducation ascendante de leurs parents, qu'ils soient les vecteurs de normes nutritionnelles et les consommateurs raisonnés de demain. Comme le montre A. Dupuy (2014) dans ses travaux sur la socialisation alimentaire contribuant à renverser les rapports de génération, l'enfant est informé et formé de manière à devenir un prescripteur susceptible de transmettre des « leçons nutritionnelles » et de modifier les habitudes alimentaires familiales. Aussi, si les adolescent(e)s nous disent avoir connu les interventions en « éducation à l'alimentation » dans le cadre de leur scolarité, ils ne semblent pas avoir été sensibles à cette intervention pédagogique sous la forme d'ateliers d' " éducation au goût » 6 .

Entre le puissant marketing social utilisé par les campagnes de promotion de la santé ; la maîtrise de soi en vue de la santé (Ibid., p. 84), et l'invitation à la consommation partagée de fast-food entre pairs, sensible au goût et à l'affirmation identitaire, on y décèle une complexité de l'alimentation contemporaine fait d'injonctions contradictoires. ; " on nous a dit souvent que c'est pas bon pour la santé mais c'est notre repère entre potes et c'est bon » (Abdel) ; «ça compte salade tomates oignons dans le kebab ? [rire] Ça fait déjà 3 ». Loin d'être anecdotique, cet usage de l'humour permet ici un contournement habile, pour ne pas dire une subversion socialement acceptée, du conformisme social (Scott, 2009), voire du conservatisme social inconscient (Larchet, 2016) auquel sont soumis les jeunes du quartier. Ici, l'humour peut contribuer à mieux absorber ce que les normes de santé ont d'incohérent, voire même d'oppressant, par l'extériorisation d'une tension qui existerait entre la pression de la conformité à une norme produite en dehors des pairs et celle produite par eux-mêmes. L'humour demeure greffé à des formes de résistance et remet en question la légitimité des normes sociales en pratiquant un écart de langage et de sens (Aird R., 2010).

$\mathrm{Au}$ fil des entretiens, cet humour laisse entrevoir des formes de résistances à ce qui serait perçue comme un "devoir citoyen à remplir » : "pourquoi genre 5 fruits et légumes ce serait mieux que de manger autre chose » (Abdel), "ça dépend de ce qui a à la cantine le jour même aussi (...) y a des jours je peux... d'autres impossible (...) D'façon on est jeune on se dépense et les légumes j’aime pas » (Mohamed). Ces verbatim marquent un scepticisme plus fort vis-à-vis de l'autorité du discours institutionnel de santé, seules les filles semblent s'accommoder du discours normatif de santé : "Le frites - kebab, pas plus d'une à deux fois par semaine » (Ibtissem) ; "il y a beaucoup de nourriture grasse dans le quartier" (Lola). En regard d'aliments ou de boissons, marqueurs forts de la qualité nutritionnelle de l'alimentation, la consommation alimentaire des jeunes filles apparaît plus favorable à la santé que celle des garçons. Comme une tendance qui s'accroit depuis quelques années déjà (Escalon et Beck, 2013), elles nous disent manger davantage de fruits et de légumes dans une journée et boire moins de sodas.

Les habitudes alimentaires sont des processus dynamiques fait de sélections, de ruptures, de continuités et de transformations. Les traditions s'alimentent des nouveautés introduites par des jeunes plus sensibles à la charge symbolique des pratiques sociales. De fait, l'alimentation est incorporée en tant que telle mais est aussi porteuse d'identité individuelle et collective placée dans un contexte culturel donné.

Les pratiques corporelles revêtent aussi des enjeux identitaires par incorporation d'un système de codes et de représentations véhiculés par des logiques internes et des mécanismes implicites (Level, Dugas et Lesage, 2010). Utilisant l'outillage théorique à Pierre Bourdieu, le système des sports élaboré s'est enrichi d'une mise en relation entre l'habitus et les modalités de pratique (Pociello, 1999) : "Les dispositions à percevoir et à agir influencent les probabilités à avoir une activité physique et sportive, et surtout la manière de pratiquer » (Vieille Marchiset, op.cit., p. 121). Tantôt relié au capital culturel et social, voire symbolique, le capital corporel et sportif est loin d'être aléatoire ou uniquement héréditaire, est également liée aux conditions de vie des familles : "les familles populaires ont toutes les chances 
d'avoir un corps plus éloigné du " corps légitime » défini par la norme médicale » (Grassler, Knobé et Gasparini, 2019).

Or, les enquêtés masculins n'ont pas attendu les messages de santé publique pour se mettre en mouvement. L'activité physique, prise au sens des messages de promotion de la santé et d'une conception santéiste et hygiénique, ne peut être comprise qu'en référence à d'autres personnes qui auraient « besoin de se mettre au sport » et, par conséquent, qui sous entendrait un problème de santé physique notamment celui de l'obésité, selon Abdel ; "Nous, on fait déjà du sport, on n'a pas besoin de faire des activités physiques, pour moi les pubs que je vois ça s'adresse pas aux jeunes, ça s'adresse pas à nous mais à ceux qui ont besoin de bouger parce qu'ils sont gros ", ou comme des activités essentiellement féminines pour Mohamed ; " pour moi l'activité physique c'est pas du sport, c'est faire du jogging, de la gym douce... des trucs de filles quoi ».

Les usages sociaux du corps classiquement attribués aux groupes sociaux les plus favorisés par une quête de forme et une recherche d'esthétique (Vieille Marchiset et Gasparini, 2010) peuvent se retrouver dans les discours des filles pour lesquelles la perte de poids reste l'une des motivations principales : "j'aimerais bien faire du Pilates ou de l'aquagym ou des trucs pour travailler mes abdos et maigrir un peu » (Lola).

Les adolescent(e)s rencontré(e)s n'ont jamais pratiqué d'activité sportive sous licence fédérale, mais prennent part à des activités organisées par la maison de quartier. Tous les mardis soirs, un tournoi de futsal inter-centre est organisé dans un complexe sportif du quartier avec les adolescents. Les mercredis après-midi, une professionnelle de la boxe vient encadrer les jeunes filles. Les jeunes se socialisent essentiellement entre pairs par des pratiques physiques qui engagent physiquement le corps dans la (re)production identitaire : "l'histoire du quartier est liée à celle du football » (Youssef). La boxe pour les adolescentes, " pour qu'elles apprennent à se défendre et parce que ça transmet des valeurs dans un quartier difficile »(Youssef). Le futsal pour les adolescents «parce que c'est le sport du quartier que tout le monde aime» (Youssef).

Alors que la promotion des activités corporelles reste marquée par un modèle biomédical (Vieille Marchiset, op.cit.), la promotion d'une alimentation saine, qui a véritablement pénétré l'espace scolaire, apparaît sous une version plus douce de la moralisation en se mouvant en " éducation au goût ». Moins touchés ou peut-être moins sensibles au marketing social de la promotion de la santé, il nous semble que les adolescents du quartier se refusent à suivre les normes produites en dehors d'euxmêmes si elles ne correspondent pas aux besoins partagés par les pairs.

\section{"Bien manger c'est le début du bonheur ! » : l'entrepreneur du bien- être entre moralisation douce et réforme des mœurs}

Bien évidemment, les catégories de publics rencontrées précédemment sont, presque, des idéaux-types de différentes fractions populaires qui nous ont permis d'identifier la distance sociale et culturelle qui les sépare des dispositifs de promotion de la santé. Il s'agit dans cette dernière partie d'adopter le point de vue d'un « entrepreneur du bien-être » (Vieille Marchiset, op. cit.) ${ }^{7}$ en faisant un focus sur une participante à un entretien collectif qui regroupe des salariés ou étudiants diplômés du supérieur ${ }^{8}$.

Madame C. est étudiante et habite le quartier depuis peu. Agée de 24 ans, elle est 
originaire de la région Poitou-Charentes, arrivée à Lille spécialement pour continuer ses études d'architecte, elle réside dans le quartier, en colocation avec d'autres étudiants. Elle s'y sent bien et y apprécie « le brassage culturel ». Elle nous explique que ses choix alimentaires sont intimement liés à son histoire personnelle, fille d'agriculteur, elle prône " un retour à la terre ». Elle est coopératrice dans un supermarché participatif du quartier qui est la plus grande association du quartier en termes de membres (+ de 1000), "la cotisation est unique et à hauteur de 100 euros (...) une fois coopérateur, on doit s'acquitter de 3 heures de bénévolats mensuels » et s'intéresse aux jardins partagés du secteur. Elle se fait la voix d'une consommatrice raisonnée qui a intériorisé, incorporé, voire surpassé, les recommandations nutritionnelles ; " bien sûr que je mange 5 fruits et légumes par jour... même plus », par l'adoption d'une consommation alternative de produits locaux et issus de l'agriculture biologique ; "plus encore que la consommation quotidienne de fruits et de légumes, il faut favoriser la production locale et les Associations pour le Maintien d'une Agriculture Paysanne (AMAP), un retour à la terre et au bio ».

Interpellée plus tard par un participant sur le coût financier des légumes biologiques, la participante répondra fermement que " ce n'est pas forcément plus cher, j'aime savoir ce que je mange ». L'union du corps et de l'esprit est primordial lors de l'acte alimentaire, le rapport à l'alimentation s'exprime de manière hédoniste : " pour moi qu'est-ce que "bien manger »? C'est le début du bonheur, c'est l'équilibre dans l'assiette, c'est manger en conscience et partager ».

Les discours laissent transparaître au mieux une culpabilisation, au pire une infantilisation des autres participants condamnés à être des sujets à éduquer : " évidemment dans le quartier ce n'est pas facile, [la rue Pierre Legrand], c'est sans doute la rue où il y a le plus de Kebabs... bien manger, ça s'apprend ". Fondamentalement, elle contribue, au regard des travaux de Norbert Elias (1991) sur le processus de civilisation des mœurs et ceux de Michel Foucault (1975 et 2004) sur la biopolitique et l'herméneutique du sujet, à une mise en discipline des comportements individuels. Autrement dit, elle entreprend une mission biopédagogique au sens de G. Rail, à savoir l'idée selon laquelle «il est possible d'abandonner un ensemble de questions historiques, sociales, environnementales, culturelles et économiques en faveur d'une simple intervention conçue pour apprendre aux gens comment vivre "de la bonne façon " (Rail, 2016, p. 26). Cette entreprise se retrouve au travers de l'explication de ses pratiques corporelles. Avant de venir dans la capitale des Flandres, elle pratiquait le handball en club depuis quelques années, elle est habituée au cadre associatif et prône « un sport collectif, fait de fraternité ».

Elle présente aux participants sa vision du sport qui correspond en tout point aux messages de santé publique et qui se retranscrit à travers un discours hygiénique, sanitaire et sécuritaire en s'opposant au discours de deux participants (Monsieur A. pratiquant de course à pied et Monsieur J. grimpeur 9 ) : «je ne pense pas qu'il faille souffrir pour avoir des résultats c'est justement pas l'objectif du sport de faire souffrir mais plutôt celui de se sentir bien dans sa peau après le sport, une bonne fatigue et un bon moment passé avec les ami(e)s ». En alliant encore une fois l'esprit et le corps dans une posture réflexive ; " être à l'écoute de son corps et de ses sensations ", elle déplore son manque de temps pour pouvoir expérimenter des pratiques corporelles de bien-être comme le yoga.

L'enquêtée distingue d'ailleurs le temps de l'activité sportive institutionnelle, de l'activité physique qui est totalement intégrée dans son quotidien, d'ailleurs son vélo est à l'entrée, et dès qu'elle le peut, elle se déplace avec, tout en faisant attention dans un quartier " où de nombreuses voitures circulent et où les pistes cyclables se comptent sur les doigts de la main ». Habituée aux démarches collectives et rompue aux pratiques participatives, elle s'exprime facilement lors de ce focus group.

Selon Pierre Bourdieu, l'imaginaire d'un corps libéré de toutes contraintes, allié à 
l'utopie sanitaire portée par la promotion de la santé, participe à la domination économique et symbolique des classes favorisées et relève d'une construction qui " attend lintégration symbolique des classes dominées, substituant la séduction à la répression, les relations publiques à la force publique, la publicité à l'autorité, la manière douce à la manière forte, attend l'intégration symbolique des classes dominées de limposition des besoins plus que de linculcation des normes » (Bourdieu, 1979, p. 72).

$\mathrm{Au}$ fond, l'« entrepreneur du bien-être » n'est-il pas «celui qui veut que les autres fassent ce que lui pense être juste » (Becker, 1985, p. 40)?

Plus ou moins consciemment, elle suggère un rapport au monde particulier en prônant une forme d'ascétisme qui se retranscrit au travers de pratiques sociocorporelles spécifiques et une posture expressive (le besoin de s'affirmer en tant que consommateur de produits locaux, biologiques et équitables) et réflexive (à la recherche d'expériences physiques et corporelles hygiéniques) de soi et sur soi. Le risque identifié ici est de réduire d'autres pratiques à ce qu'elles paraissent incohérentes, voire inacceptables, au regard des normes sanitaires et sociales, au regard même de l'environnement.

\section{Conclusion}

Les dispositifs de promotion de la santé laissent une place centrale à la question morale de la gestion individualisée et rationalisée des corps. En guidant les corps et les consciences vers un « savoir-vivre » mais aussi un "savoir-être », une éthique de l'(auto)-responsabilité est ainsi recherchée par un devoir de "gouvernement de soi ». Si cette étude donne à voir certains décalages socio-culturels qu'il existe au travers des représentations de la santé conditionnées par des rapports au monde spécifiques, l'éducation à la santé ne peut être réduite à une institution de domination tant les problèmes de santé publique peuvent s'additionner dans les territoires qui cumulent les difficultés.

Elle peut même entrer en contradiction avec les profits économiques des acteurs agro-alimentaires, industriels mais aussi avec l'environnement socio-écologique des sujets enquêtés. C'est alors que les processus normatifs antagonistes et les injonctions contradictoires entrent en jeu et fonctionnent à plein régime dans l'espace urbain.

Cela dit, notre dernière focale qui intègre la variable spatiale nous invite à considérer l'espace urbain non seulement comme un " environnement supportif » à la santé et au bien-être (Charte d'Ottawa, 1986), mais aussi et surtout, comme un instrument de gouvernement de la mobilité des hommes (Béal et Rousseau, 2008). Il incite à la fréquentation quotidienne pour ses activités socialisantes qui deviennent autant de supports d'une " surveillance naturelle » (Tozzi, 2013) revenant à envisager de discipliner les espaces apparemment «non disciplinaires » (Foucault, 1975). Le quartier populaire en est un exemple.

Parallèlement, c'est donc aussi au travers d'un discours teinté de "prévention situationnelle » que l'on assiste à l'intégration de la démarche environnementale et hygiéniste dans la promotion de la santé où chaque " éco-citoyen » est incité à adopter un comportement responsable, de sa santé a minima. Ce militantisme d'un " esprit sain » dans un " corps sain », d'ascèse corporelle en somme, ne nous replonge-t-il pas dans l'espace théorique du naturisme de l'entre-deux guerres où penser un mode de vie autre, plus sain, plus libéral était la condition de possibilité de l'émancipation (Riondet et Go, 2017) ? Par l'imposition de nouveaux besoins alimentaires et de nouvelles normes de pratiques corporelles, ne voit-on pas ici l'émergence d'un biopouvoir qui puisse servir certains groupes sociaux ou politiques ? La notion de démocratie sanitaire n'intègre-t-elle pas, de fait, les mêmes franges de population dans les démarches participatives en éducation à la santé ? Si tel est le cas, on peut aisément imaginer qu'une économie de l'embourgeoisement urbain se mette en place autour des projets de promotion de la santé. 


\section{Bibliographie}

Aceti, M., \& Vieille Marchiset, G. (2014). Quatre programmes nationaux à la loupe en Europe, (140), 41-45.

Béal, V., \& Rousseau, M. (2008). Néolibéraliser la ville fordiste. Politiques urbaines postkeynésiennes et re-développement économique au Royaume-Uni : une approche comparative. Métropoles [En ligne], 4 | 2008, mis en ligne le 18 décembre 2008. URL : http://journals.openedition.org/metropoles/3502; DOI : $\quad$ https://doi.org/10.4000 /metropoles.3502

Becker, H. S. (1985). Outsiders. Etudes de sociologie de la déviance. Paris : Métailié.

Belbachir, S., Bienvenu, C., Délé, C. B., Duchaussoy, G., \& Rahmania, M.-R. (2016). Vivre ensemble à Lille-Fives. Des liens sociaux à l'épreuve d'un territoire en mutation. In M. Carrel, P. Cary et J.-M. Wachsberger (dir.), Ségrégation et fragmentation dans les métropoles : Perspectives internationales (pp. 323-344). Lille : Presses Universitaires du Septentrion.

Berlivet, L. (2013). Les ressorts de la « biopolitique » : « dispositifs de sécurité » et processus de « subjectivation» au prisme de l'histoire de la santé. Revue d'histoire moderne contemporaine, 6o-4/4 bis (4), 97-121.

Berlivet, L. (2015). Une biopolitique de l'éducation pour la santé : La fabrique des campagnes de prévention. In D. Fassin et D. Memmi (dir.), Le gouvernement des corps (pp. 37-75). Paris : Éditions de l'EHESS.

Boltanski, L. (1971). Les usages sociaux du corps. Annales ESC, 26/1, 205-233.

DOI : $10.3406 /$ ahess.1971.422470

Bossy, T., \& Briatte, F. (2011). Les formes contemporaines de la biopolitique. Revue internationale de politique comparée, vol. 1/4, 7-12.

DOI : 10.3917/ripc.184.0007

Bourdelais, P. (2001). Les hygiénistes : enjeux, modèles et pratiques, XVIIIe-XXe siècles. Paris : Belin.

Bourdieu, P. (1979). La Distinction : Critique sociale du jugement. Paris : Minuit.

Bourdieu, P. (1987). Choses dites. Paris : Minuit.

Corbeau, J.-P., \& Poulain, J.-P. (2002). Penser l'alimentation. Entre imaginaire et rationalité. Toulouse : Privat.

Cresson, G. (1997). La sociologie de la médecine méconnaît-elle la famille ? Sociétés Contemporaines, 25(1), 45-65.

DOI : $10.3406 /$ socco.1997.1434

Dardot, P., \& Laval, C. (2009). La nouvelle raison du monde. Essai sur la société néolibérale. Paris : La Découverte.

Darmon, M. (2007). La socialisation. Paris : Armand Colin.

Dozon, J.-P., \& Fassin, D. (2001). Critique de la santé publique : Une approche anthropologique. Paris : Balland.

Dupuy, A. (2014). Regard(s) « sur» et « par» l'alimentation pour renverser et comprendre comment sont renversés les rapports de générations : l'exemple de la socialisation alimentaire inversée. Enfances Familles Générations. Revue interdisciplinaire sur la famille contemporaine, $20,79-108$.

Dupuy, A., \& Poulain, J.-P. (2008). Le plaisir dans la socialisation alimentaire. Enfance, vol. $60 / 3,261-270$.

DOI : $10.3917 /$ enf.603.0261

Elias, N. (1991, 1ère ed. all. 1939). La civilisation des mours. Paris : Calmann-Lévy.

Escalon, H., \& Beck, F. (2013). Les jeunes et l'alimentation. Agora débats/jeunesses, 63/1, $113-127$.

Fassin, D. (1990). Décrire. Entretien et observation. Paris : Ellipses.

Fassin, D. (1996). L'espace politique de la santé : Essai de généalogie. Paris : PUF.

DOI : 10.1016/S1240-1307(97)86228-0

Fauquette, A., \& Gibout, C. (2019a). « Couvrez ce kébab que je ne saurais voir !» : alimentation alternative ou nouvelle gouvernementalité des corps ? Communication présentée au Séminaire européen "Alimentation, régénération urbaine et développement durable des territoires en Europe », Lille : Maison Européenne des Sciences de l'Homme et de la Société.

Fauquette, A., \& Gibout, C. (2019b). Le « bien manger »: une arme de consentement massif pour repenser le territoire. Communication présentée au 15ème Congrès de l'Association Française de Science Politique (ST 49 : Politique de l'alimentation et territoires, politique des 
territoires et alimentation), Bordeaux : Institut d'Etudes Politiques.

Faure, S. (2008). Les politiques du corps visant les milieux populaires. Lien social et Politiques, 59, 33-45.

Faure, S., \& Thin, D. (2019). S'en sortir malgré tout : Parcours en classes populaires. Paris : La Dispute.

Foucault, M. (1961). Folie et déraison. Histoire de la folie à l'âge classique. Paris : Plon.

Foucault, M. (1975). Surveiller et punir. Naissance de la prison. Paris : Gallimard.

Foucault, M. (2001). L’Herméneutique du sujet : Cours au Collège de France. Paris : Seuil.

Foucault, M. (2004). Naissance de la biopolitique. Cours au Collège de France, 1978 - 1979. Paris : EHESS, Gallimard, Seuil.

Génolini, J.-P., \& Clément, J.-P. (2010). Lutter contre la sédentarité : L'incorporation d'une nouvelle morale de l'effort. Sciences sociales et sport, 3/1, 133-156.

DOI : $10.3917 /$ rsss.003.0133

Gibout, C. (2000). Voyage au bout de la Nuitée. Approche sociologique de la population féminine fréquentant le foyer d'urgence pour sans-abris à Tours (France). In A. Neculau \& G. Ferréol (eds.). Aspecte psihosociale ale saraciei - Psycho-Sociologie de la Pauveté. (pp. 216-226). Bucarest : Poliram.

Grafmeyer, Y., Joseph, I. (Dir.). (2009). L'Ecole de Chicago : Naissance de l'écologie urbaine. Paris : Flammarion.

Grassler, M., Knobé, S., \& Gasparini, W. (2019). Contrôle du poids des enfants et activité physique dans un quartier populaire strasbourgeois : modèles familiaux et recommandations de santé publique. Enfances Familles Générations. Revue interdisciplinaire sur la famille contemporaine, $3,75-88$.

DOI : $10.7202 / 1067812 \mathrm{ar}$

Guinchard, C. (2011). Logiques du dénuement, Réflexions sociologiques sur la pauvreté et le temps. Paris: L'Harmattan.

Gusfield, J. (1963). Symbolic Crusade. Status Politics And The American Tempearance Movement. Champaign : University of Illinois Press.

Hoggart, R. (1970). La Culture du Pauvre : Etude sur le style de vie des classes populaires en Angleterre. Paris : Minuit.

Koch, E. R. (2013). La pratique du goût : de Pierre Bourdieu à Antoine de Courtin. Dixseptième siècle, 258/1, 45-54.

Lamy, J. (2012). La fabrique politique du corps : historiographie sélective des héritages foucaldiens. Cahiers d'histoire. Revue d'histoire critique, 118, 91-114

DOI : $10.4000 /$ chrhc. 2526

Larchet, N. (2016). Changer les habitudes ou changer les habitants ? Sociologie d'un mouvement de réforme alimentaire à la Nouvelle-Orléans. 2000-2010. Thèse de Doctorat. Paris : EHESS.

Le Breton, D. (2010). L'obésité entre dissidence et résistance. In H. Bergeron, P. Castel (Dir.), Regards croisés sur l'obésité (pp. 19-28), Paris : Editions de santé et Presses de Sciences Po.

Level, M., Dugas, É., \& Lesage, T. (2010). Jeux sportifs, codes et construction identitaire : règles explicites des mécanismes internes et règles implicites du pratiquant. Ethnologies, 32/1, $113-132$.

Louveau, C. (2007). Le corps sportif : un capital rentable pour tous ?, Actuel Marx, 41/1, $55^{-70}$.

DOI : 10.3917/amx.041.0055

Masy, J. (2013). La temporalité, une disposition sociale et culturelle de construction de l'avenir. Le cas des « aspirants » aux classes préparatoires aux grandes écoles. SociologieS, 1-17.

Maurice, A. (2014, 24 septembre). Les préadolescents comme ressorts des actions de santé publique : analyse d'un projet d'éducation alimentaire en collège. Thèse de doctorat, Paris : Université Paris Descartes.

Memmi, D., \& Taïeb, E. (2009). Les recompositions du « faire mourir » : vers une biopolitique d'institution. Sociétés Contemporaines, 75/3, 5-15.

Millet, M., \& Thin, D. (2005). Le temps des familles populaires à l'épreuve de la précarité. Lien social et Politiques, 54, 153-162.

Parayre, S. (2010). L'entrée de l'éducation à la santé à l'école par la prévention (XVIIIe-XIXe siècles) : Protéger, renforcer et redresser le corps. Recherches \& éducations, 3, 25-46.

Peretti-Watel, P., \& Moatti, J.-P. (2009). Le principe de prévention. Le culte de la santé et ses 
dérives. Paris : Seuil.

Pierret, J. (1984). " Les significations sociales de la santé. Paris, l'Essonne, l'Hérault ». In M. Augé \& C. Herzlich (Dir.). Le sens du mal. Anthropologie, histoire et sociologie de la maladie (pp 217-256). Paris : Éditions des Archives Contemporaines.


sportifs, Paris : PUF.

Rail, G. (2016). La violence de l'impératif du bien-être. Bio-Autres, missions de sauvetage et justice sociale. Staps, 112(2), 17-31.

Riondet, X., \& Go, H. L. (2017). Le corps chez les éducateurs marxo-naturistes pendant l'Entredeux-guerres. Une écologie corporelle et une politique de l'ordinaire ? Corps, 15/1, 45-55.

DOI : 10.3917/corp1.015.0045

Rousseau, M. (2008). La ville comme machine à mobilité. Capitalisme, urbanisme et gouvernement des corps. Métropoles [En ligne], 3 | 2008, mis en ligne le 12 septembre 2008. URL : http://journals.openedition.org/metropoles/2562

Schwartz, O. (1998). La notion de «classes populaires ». Mémoire pour l'habilitation à diriger des recherches. Versailles : Université de Versailles - Saint - Quentin.

Scott, J. C. (2009). La Domination et les arts de la résistance : Fragments du discours subalterne. Paris : Amsterdam.

Séchet, R. (2006). Le populaire et la saleté : de l'hygiénisme au nettoyage au Karcher. In, Th. Bulot \& V. Veschambre (Dir.). Mots, traces et marques (dimensions spatiale et linguistique de la mémoire urbaine) (pp. 246 et al.). Paris : L'Harmattan.

Seignan, G. (2010). L'hygiène sociale au XIXe siècle : une physiologie morale. Revue d'histoire du XIXe siècle, 40, 113-130.

DOI : $10.4000 /$ rh19.3996

Silk, M. L., \& Andrews, D. L. (2011). Toward a Physical Cultural Studies. Sociology of Sport Journal, 28/1, 4-35.

DOI : $10.1123 / \operatorname{ssj} .28 .1 .4$

Thin, D. (1997). "Tant qu'on a la santé » Des familles populaires et de la santé de leurs enfants. Rapport d'étude sociologique réalisé pour le groupe " Action santé sociale pilote » de Bron Parilly. Lyon : Université Lumière Lyon 2.

Thin, D. (2009). Un travail parental sous tension : les pratiques des familles populaires à l'épreuve des logiques scolaires. Informations sociales, 154/4, 70-76.

DOI : $10.3917 /$ inso.154.0070

Tozzi, P. (2013). Ville durable et marqueurs d'un « néo-hygiénisme » ? Analyse des discours de projets d'écoquartiers français. Norois. Environnement, aménagement, société, 227, 97-113.

Turner, B. S. (1982). The Governement of the Body: Medical Regimens and the Rationalization of Diet, The British Journal of Sociology, 3/2, 254-269.

Vieille Marchiset, G. (2019). La conversion des corps Bouger pour être sain. Paris : L'Harmattan.

Vieille Marchiset, G., \& Aceti, M. (2013). La fabrique des normes corporelles dans les programmes de promotion de santé par les activités physiques en rupture avec les quartiers pauvres : une comparaison européenne. Communication au Congrès de la 3SLF « La Comparaison en Sciences du Sport ». Strasbourg : Université de Strasbourg.

Vieille Marchiset, G., \& Gasparini, W. (2010). Les loisirs sportifs dans les quartiers populaires : modalités de pratiques et rapports au corps. Staps, 87/1, 93-107.

\section{Notes}

1 Lancé en 2001, le Programme national nutrition santé (PNNS) est un plan de santé publique visant à améliorer l'état de santé de la population en agissant sur l'un de ses déterminants majeurs : la nutrition. Pour le PNNS, la nutrition s'entend comme l'équilibre entre les apports liés à l'alimentation et les dépenses occasionnées par l'activité physique. Ce programme est inscrit dans le code de la santé publique (article L 3231-1) comme un programme quinquennal gouvernemental. PNNS1 (2001-2005) ; PNNS2 (2006-2010) ; PNNS3 (2011-2016) ; PNNS 4 (2019-2023).

2 A. Fauquette et C. Gibout ont enquêté sur « l'alimentation alternative » et le « bien manger " dans le quartier où nous enquêtons. Leurs recherches, en cours de valorisation, ont fait l'objet de plusieurs communications (Fauquette et Gibout, 2019a et 2019b) et d'articles actuellement en navettes d'expertise scientifique. 
3 Même si l'un des auteurs vit dans le quartier, originellement (avant les 3 années de l'étude) il était peu investi dans la vie sociale locale.

4 La notion d'« entrepreneur du bien-être » est mobilisée par plusieurs auteurs sous différentes formes et en référence aux «Symbolic Crusade » de J. Gusfield (1963) et aux " entrepreneurs de la morale » chez H. S.Becker (1985, pp. 171-186). Dans notre contexte scientifique : les « entrepreneurs du bien-être corporel » chez Gilles Vieille Marchiset dans une communication scientifique présentée aux journées "La santé en corps » à l'Université Paris Descartes (Juin 2018) et qui réapparaît dans son ouvrage précédemment cité (Vieille Marchiset, 2019, pp. 69-101).

5 Le « wagon » est un wagon désaffecté transformé en un lieu de restauration pour les plus démunis qui est situé derrière la gare Saint Sauveur à Lille, à quelques encablures du quartier enquêté. Des repas y sont distribués par les bénévoles de l'association "Les Restos du cœur ».

6 Ce scepticisme à l'égard des interventions pédagogiques scolaires a fait l'objet d'un travail de thèse. Celui d'A. Maurice (2014) qui s'est appliquée à démontrer que les populations défavorisées sont moins perméables aux informations véhiculées par les programmes nationaux de santé. Elle met en lumière des " messagers » essentiellement issus de milieux favorisés.

7 La méthode choisie est peu utilisée jusqu'alors. Généralement, il s'agit de confronter les discours normatifs et injonctifs produits par les acteurs institutionnels de la promotion de la santé dans le cadre de programme d'éducation à la santé à des individualités (ce qui demande un travail quantitatif considérable). Or, notre travail ici consiste à montrer qu'il existe des acteurs individuels, des citoyens " entrepreneurs de santé (du bien-être) » qui agissent au travers de démarches participatives et qui participent informellement ou formellement à une « éducation à la santé au quotidien » interne au quartier.

8 Le choix du lieu où se déroule l'entretien collectif n'est pas anodin. Il nous a permis d'avoir accès à une population du même quartier mais avec d'autres caractéristiques sociales et culturelles. Rappelons-le, il s'agit de l'« avant-goût de la cuisine commune », un nouveau lieu qui intervient dans le processus de transformation et de renouveau du quartier de Fives.

9 Nous retrouvons dans cet entretien collectif les caractéristiques des personnes capitalisant les ressources sociales et culturelles en tant que pratiquants réguliers d'activités physiques et sportives.

\section{Pour citer cet article}

Référence électronique

Quentin Courcier, Florian Lebreton et Christophe Gibout, « L'éducation à la santé

ethnographiée dans un quartier populaire:

entre conformisme et dissidence », Recherches \& éducations [En ligne], 22 | 2021, mis en

ligne le , consulté le 03 mars 2021. URL : http://journals.openedition.org/rechercheseducations

/11163 ; DOI : https://doi.org/10.4000/rechercheseducations.11163

\section{Auteurs}

Quentin Courcier

ULCO

TVES UR4477

Florian Lebreton

ULCO

TVES UR4477

Christophe Gibout

ULCO

TVES UR4477

\section{Droits d'auteur}

(C) Tous droits réservés 